\title{
Del texto al discurso: una propuesta de análisis para abordar el componente textual en canciones con estructura narrativa
}

\author{
Danis Eduardo Ruíz Toro ${ }^{1}$ \\ Fundación Universitaria Tecnológico Comfenalco
}

\section{Resumen}

En el trabajo que presentamos a continuación exponemos una propuesta metodológica para analizar el componente textual de canciones con estructura narrativa. La pretensión de esta forma de análisis es tomar como base la estructura temática, la estructura informativa y la estructura genérica de las letras de las canciones con el fin analizar 1) las herramientas de cohesión y de coherencia (elipsis, referencias anafóricas y exofóricas, deixis, relaciones léxicas, relaciones de causa-efecto, causa-consecuencia, etc.), y 2) las implicaciones discursivas presentes en la complejidad semiótica de distintos aires musicales que se expresan en el componente textual de las canciones. Este método de análisis se apoya en la Lingüística Sistémico-Funcional (LSF), propuesta por Halliday (2004), y se complementa con los aportes

\section{Abstract}

This paper exposes one methodology proposal to analyze the textual components of songs and their narrative structures. This type of analysis pretends to study three basic structures: thematic, informative and generic structures of lyrics to observe 1) cohesion and coherence tools (ellipsis, anaphoric and exophoric references, deictics, lexical relations, cause and effect relations, cause and consequence, etc.) and 2) the discursive implications presents at semiotic complexity of different musical styles which are reflected in the textual component of songs. This method of analysis in theory is based on Systemic Functional Linguistics (SFL), proposed by $M$. A. K. Halliday (2004) and complemented by the contributions of authors as Barthes (1977)

\footnotetext{
${ }^{1}$ Magíster en Lingüística de la Universidad Nacional de Colombia. Docente de la Fundación Universitaria Tecnológico Comfenalco. e-mail: danisicfes2007@hotmail.com
}

Recibido: Marzo, 2010 - Aprobado: Junio, 2010. 
de autores como Barthes (1977) y Paz Gago (1989). Los ejemplos que se muestran giran alrededor de las primeras cláusulas de la canción "Camino a la delincuencia", interpretada por el cantante cartagenero Papomán.

Palabras clave: componente textual, conexión informativa, estructura genérica, estructura informativa, estructura temática. and Paz Gago (1989). The examples are shown to explain the method is centered on the first clauses of song "Camino a la delincuencia", starring Papomán.

Key words: textual component, informative connections, thematic structures, informative structures, generic structures.

\section{Introducción}

Como fenómenos culturales, los aires musicales son de interés para muchas ciencias humanas y económicas desde las cuales pueden analizarse cómo ciertos aspectos de la vida cotidiana se integran semióticamente en su complejo significativo. Para nadie es un secreto que el hecho de que la música haga parte de la cultura implique que las canciones presenten una carga ideológica y social sin la que muchos de sus sentidos resultarían incomprensibles. No obstante, lo que puede interesar de este conocimiento común es el hecho de suponer que si los mismos aspectos ideológicos se descubren, tomando como referente el componente textual de las canciones propias de un aire musical, se necesita un método que permita analizarlo para descubrir las dimensiones lingüísticas que evidencian matices de la cultura.

Tal necesidad se comprende si, por un lado, se hace consciencia de que es en el texto -letras de las canciones- donde se visibilizan las interpretaciones que los actores sociales hacen de los temas universales que les interesan, y por otro lado, si se tienen en cuenta las siguientes preguntas: ¿Cómo se organiza el mensaje en el componente textual de los textos de las canciones? ¿Qué lecturas sobre los temas universales y sus participantes se muestran a partir de la textura (sistema temático, informativo y estructura genérica) de las canciones? Para la elaboración de esta propuesta de análisis hemos tomado como base ciertas implicaciones teóricas dentro del enfoque de la Lingüística Sistémica Funcional de M. A. K Halliday, en la que se entiende el componente textual como aquel que incluye los recursos del sistema lingüístico, específicamente los creadores de texto, esto es, el sistema temático, el sistema de información, los elementos de cohesión interna y la estructura genérica. Para Halliday estos recursos componen la textualidad y son suficientes para definir y analizar un texto (1985:174-8). 
De igual forma, en el enfoque sistémico se afirma que el sistema temático se refiere a la forma cómo se organiza la información en las cláusulas, donde una parte es enunciada como tema y la otra como rema. La primera funciona como punto de partida, o entidad con la que resulta pertinente expresar la predicación que le sigue, y la segunda, como la predicación que se desarrolla en la secuencia de significado (2004: 64-5). El sistema de información, según Halliday, está compuesto por unidades de información. Es decir, por estructuras configuradas por dos funciones: lo Nuevo y lo Dado. La información "nueva" es aquella que se supone no es conocida por el oyente, porque no se ha mencionado en la situación, y la información vieja es aquella que ya se ha mencionado (89). Por último, la estructura genérica se refiere a la forma que posee un texto como una propiedad de su género. En otras palabras, a la estructura predominante que posee como muestra de una tipología textual: narrativa, argumentativa, expositiva o descriptiva.

Para ampliar teóricamente la información sobre la estructura genérica, tomamos como referente a Roland Barthes (1977), quien introduce los siguientes términos: indicios caracterológicos, eventos funcional-cardinales y eventos funcional-catalizadores. Los primeros son aquellos que caracterizan a participantes y circunstancias espacio-temporales, e implican eventos que sucederán alrededor de ellos -por esta razón, para entenderlos, hay que pasar al nivel de las funciones (Barthes, 1977: 20-21)-. Los eventos funcionalcardinales son aquellos que abren una alternativa consecuente para la continuación de la historia, por lo que incluyen el nodo de información con sus relaciones semánticas. Los eventos funcionalcatalizadores son aquellos que complementan informativamente a los eventos funcional-cardinales, por lo que incluyen relaciones lógicas y semánticas que nutren los nodos de información (20-1).

Con el apoyo de los presupuestos teóricos mencionados, explicaremos, en primer lugar, la organización de las tablas de análisis de las estructuras que configuran la textualidad de las canciones. En segundo lugar, presentaremos la forma cómo se interpretan los datos, y por último, haremos una conclusión reflexiva en torno a los resultados que se pretenden alcanzar con la aplicación de este método de análisis.

\section{Organización de las tablas de análisis de las estructuras}

Dado que son tres estructuras o sistemas individuales interdependientes, organizaremos una tabla para cada uno de ellos y haremos 
la descripción pertinente. Así, en la temática describiremos lo que se tematiza y los recursos lingüísticos empleados para ello; en la informativa, lo que se presenta como nuevo y los recursos no estructurales con que se expresa lo noticioso; y en la genérica, las características de la configuración estructural del texto como muestra de una tipología textual definida. Procederemos, en cada una de las tablas, a dividir la canción en la unidad de análisis de la Gramática Sistémico Funcional. Es decir, en cláusulas como unidades gramaticales que expresan un significado en una secuencia y que son construidas por grupos de palabras con un sujeto y un verbo (Halliday, 2004: 8). Tal división puede ser tanto en cláusulas simples como en complejos de cláusulas (conjunto de cláusulas simples unido gramaticalmente por un nexo de cláusula), porque no se puede perder la secuencia de significado, y si los complejos de cláusulas se rompen en cláusulas simples es posible que el sentido del mismo se vea truncado.

\subsection{Tabla de análisis del sistema temático}

La tabla de análisis del sistema temático presenta la siguiente forma:

\begin{tabular}{|c|c|c|c|c|c|c|}
\hline \multirow{2}{*}{$\begin{array}{c}\text { Intér- } \\
\text { prete }\end{array}$} & Textura & \multicolumn{4}{|c|}{ Título del texto } & $\begin{array}{c}\text { Realida- } \\
\text { des del } \\
\text { univer- } \\
\text { so }\end{array}$ \\
\cline { 2 - 7 } & $\begin{array}{c}\text { Estruc- } \\
\text { tura te- } \\
\text { mática }\end{array}$ & $\begin{array}{c}\text { Conjun- } \\
\text { ción }\end{array}$ & Elipsis & $\begin{array}{c}\text { Refe- } \\
\text { rencia }\end{array}$ & $\begin{array}{c}\text { Cohe- } \\
\text { sión } \\
\text { léxica }\end{array}$ & $\begin{array}{c}\text { Temati- } \\
\text { zada }\end{array}$ \\
\hline
\end{tabular}

En la primera columna de la tabla aparece el término "Intérprete" o "Autor", seguido del nombre del mismo. La decisión depende de las lógicas de producción del aire musical en cuestión. Por ejemplo, en el caso de la champeta el cantante es casi siempre el autor de la canción y el concepto de derecho de autor está más ligado al pickup que compra los derechos y a los cantantes, que a quien compone las canciones. Debajo de esta columna transcribiremos el texto en cláusulas simples y en complejos de cláusulas, pues es en éstas donde se sombreará en negrilla aquella palabra o grupo de palabras que represente lo tematizado. En la misma fila del término "Intérprete" se coloca "Título de la canción", como nombre que identifica la tabla de análisis. Debajo de éste, se ubican tres columnas: "Textura", "Cohesión interna" y "Realidades del universo". 
En la columna "Textura" se anota el término "Estructura temática", donde se señala el tipo de tema que se construye y la palabra o grupo de palabras que lo expresa. Cuando se hace referencia al tipo de tema, se alude a si es textual o tópico. El primero consiste en que la tematización se da por elementos estructurales como conjunciones, conectores o deícticos, y apunta al orden o forma de presentación del discurso. El segundo consiste en que lo que se tematiza dialoga con la realidad experiencial del compositor de la canción, es decir, con los procesos, participantes y factores circunstanciales: espacio, tiempo, herramientas, etc. (Halliday, 2004: 79).

Por otra parte, en la columna "Cohesión interna" se describen los recursos lingüísticos (estructurales) con que se tematiza en las cláusulas, pues como Halliday afirma: "al texto podemos caracterizarlo con referencia al sistema, en relación con la selección de opciones semánticas desplegadas a través del texto" (26). Con base en esta afirmación, la columna se subdivide en cuatro columnas: "Conjunción”, “Elipsis", “Referencia” y “Cohesión léxica”, pero en el análisis sólo se señalará aquel recurso lingüístico que expresa el tema de la cláusula, y entre corchetes se anotará la palabra o grupo de palabras que lo representa. Veamos un ejemplo:

\begin{tabular}{|c|c|c|c|c|c|c|}
\hline \multirow{2}{*}{ Intérprete } & \multicolumn{5}{|c|}{ Camino a la delincuencia } \\
\cline { 2 - 6 } & Textura & \multicolumn{4}{|c|}{ Cohesión Interna } & $\begin{array}{c}\text { Realida- } \\
\text { des del } \\
\text { univer- } \\
\text { so }\end{array}$ \\
\cline { 2 - 6 } & $\begin{array}{c}\text { Estruc- } \\
\text { tura } \\
\text { temá- } \\
\text { tica }\end{array}$ & $\begin{array}{c}\text { Con- } \\
\text { junción }\end{array}$ & Elipsis & $\begin{array}{c}\text { Refe- } \\
\text { rencia }\end{array}$ & $\begin{array}{c}\text { Cohe- } \\
\text { sión } \\
\text { léxica }\end{array}$ & $\begin{array}{c}\text { Temati- } \\
\text { zada }\end{array}$ \\
\hline $\begin{array}{c}\text { Esta es la } \\
\text { historia } \\
\text { de un mal } \\
\text { padre que } \\
\text { abandonó a } \\
\text { una niñita }\end{array}$ & $\begin{array}{c}\text { Textual } \\
\text { Thisto- } \\
\text { ria...] }\end{array}$ & & & $\begin{array}{c}\text { Dejeto } \\
\text { de ca- } \\
\text { recteri- } \\
\text { zación } \\
\text { [historia } \\
\text { de un } \\
\text { mal } \\
\text { padre] }\end{array}$ \\
\hline
\end{tabular}

Podemos observar que lo tematizado es la historia (por eso se encierra en corchetes esta palabra en la columna "Estructura temática") e igualmente, que el recurso de cohesión empleado es un elemento estructural, precisamente un deíctico demostrativo (por ello es un tema textual). Por último, la columna "Realidades del universo" se refiere al aspecto del mundo experiencial que se ha tematizado (si es un objeto, un participante, un evento o una circunstancia espacial o temporal). Observemos nuevamente el cuadro y notemos que lo que 
ha sido tematizado es la historia como objeto posible de caracterizar. Pues no es cualquier historia, sino la de un mal padre. Veamos a continuación la forma de analizar el sistema informativo.

\subsection{Análisis del sistema informativo}

La tabla de análisis del Sistema Informativo tuvo la siguiente forma:

\begin{tabular}{|c|c|c|c|c|c|c|}
\hline \multirow{4}{*}{$\begin{array}{c}\text { Intér- } \\
\text { prete }\end{array}$} & Textura & \multicolumn{4}{|c|}{ Título del texto } & $\begin{array}{c}\text { Herra- } \\
\text { mientas } \\
\text { de co- } \\
\text { hesión }\end{array}$ \\
\cline { 2 - 6 } & $\begin{array}{c}\text { Estruc- } \\
\text { tura } \\
\text { infor- } \\
\text { mativa }\end{array}$ & $\begin{array}{c}\text { Causa- } \\
\text { Motivo }\end{array}$ & $\begin{array}{c}\text { Conse- } \\
\text { cuencia }\end{array}$ & Efecto & $\begin{array}{c}\text { Fina- } \\
\text { lidad- } \\
\text { Propó- } \\
\text { sito }\end{array}$ & $\begin{array}{c}\text { Cohe- } \\
\text { sión }\end{array}$ \\
\hline
\end{tabular}

Los aspectos concernientes a la mención del nombre del intérprete y el título del texto continúan iguales que en la anterior tabla, pero las variaciones se dan en el análisis. Por una parte, en la columna "Textura" se cambia el término "Estructura temática" por "Estructura informativa", para hacer referencia al aspecto presentado como nuevo (noticia). Por otra parte, donde se ubicaba el término "Cohesión interna" se anota la expresión "Coherencia lineal", entendida como las relaciones semánticas entre oraciones (cláusulas) individuales de una secuencia. Es decir, de una ordenación lineal de oraciones en el tiempo o en el espacio (Van Dijk, 1996: 25).

El análisis desde este concepto implica entender el sistema informativo en funciones semánticas, pues como la unidad básica de la semántica es el texto, la gramática construye otros recursos no estructurales para organizar el flujo del discurso y crear contactos semánticos entre las cláusulas, los cuales trabajan bien dentro o entre las cláusulas (Halliday, 2004: 87). Ahora bien, lo que se describe son las relaciones semánticas que permiten que, desde lo informativo, el texto sea coherente, pues esto es básico en la idea de textura como unidad. Así pues, los recursos no estructurales que se analizan como subcolumnas son relaciones de "Causa-Motivo", "Consecuencia", "Efecto" y "Finalidad-Propósito", en los cuales sólo se rellena la nueva información. Veamos: 


\begin{tabular}{|c|c|c|c|c|c|c|}
\hline \multirow{2}{*}{ Intérprete } & Textura & \multicolumn{4}{|c|}{ Título del texto } & $\begin{array}{c}\text { Coherencia lineal } \\
\text { mientas } \\
\text { de co- } \\
\text { hesión }\end{array}$ \\
\cline { 2 - 6 } & $\begin{array}{c}\text { Estruc- } \\
\text { tura } \\
\text { infor- } \\
\text { mativa }\end{array}$ & $\begin{array}{c}\text { Causa- } \\
\text { Motivo }\end{array}$ & $\begin{array}{c}\text { Con- } \\
\text { se- } \\
\text { cuen- } \\
\text { cia }\end{array}$ & Efecto & $\begin{array}{c}\text { Fina- } \\
\text { lidad- } \\
\text { Pro- } \\
\text { pósi- } \\
\text { to }\end{array}$ & $\begin{array}{c}\text { Cohe- } \\
\text { sión }\end{array}$ \\
\hline $\begin{array}{c}\text { Esta es la } \\
\text { historia de un } \\
\text { mal padre que } \\
\text { abandonó a } \\
\text { una niñita }\end{array}$ & $\begin{array}{c}\text { Nuevo, } \\
\text { proceso } \\
\text { [aban- } \\
\text { dono... }\end{array}$ & $\begin{array}{c}\text { Causa } \\
\text { de efecto } \\
\text { [quedón } \\
\text { solita] }\end{array}$ & & & & \\
\hline $\begin{array}{c}\text { Y ésta se } \\
\text { quedó solita } \\
\text { acompañan- } \\
\text { do a la madre } \\
\begin{array}{c}\text { Dando gritos } \\
\text { de descuadre }\end{array}\end{array}$ & $\begin{array}{c}\text { Nuevo, } \\
\text { estado } \\
\text { [quedó } \\
\text { solita] }\end{array}$ & & & $\begin{array}{c}\text { Efecto de } \\
\text { proceso } \\
\text { [aban- } \\
\text { donó] }\end{array}$ & & $\begin{array}{c}\text { Conjun- } \\
\text { ción, ex- } \\
\text { tensión, } \\
\text { aditiva } \\
\text { [y] }\end{array}$ \\
\hline
\end{tabular}

El análisis del sistema informativo en ningún momento debe olvidar el sistema temático, pues aunque son diferentes (porque el informativo puede estar al nivel de la cláusula o sobrepasarlo), lo que se informa guarda relación lógica con lo que se tematiza. Por lo mismo se entiende que, en el ejemplo anterior, el primer complejo de cláusulas expone como información nueva el proceso ejecutado por el padre, que (a través de una trasposición adjetiva "de un mal padre") caracteriza el objeto historia, y en esa medida es la causa del efecto que se expresa en el complejo de cláusulas siguiente. Cuando hay una relación lógica entre una cláusula y otra, se anota entre corchetes la palabra o grupo de palabras con las que se guarda la relación. Por ejemplo, se puede advertir que en el primer complejo se registra que ese proceso es causa de efecto del estado: “[quedó solita]", y en el segundo complejo de cláusulas se anota que ese estado es efecto del proceso anterior: "[abandonó]".

Por último, la columna "Cohesión interna" se refiere a aquellos elementos estructurales que ayudan cohesivamente a la construcción de las relaciones semánticas y a la presentación de informaciones nuevas, lo que quiere decir que, si bien es cierto que las informaciones se construyen a partir de procesos semánticos, también es cierto que para la organización de los mismos se necesitan las herramientas de cohesión para ordenarlos y convertir el texto en una unidad. Al respecto, Halliday afirma: "en el habla es evidente que los recursos fonológicos son bien marcados en la expresión de nuevas informaciones, pero en el ámbito de lo escrito no se cuenta con este 
aspecto, por lo cual hay que recurrir a elementos de la expresión escrita (elipsis, conectores, correferencias, etc.)" (1982: 175).

Para cerrar este aparte, entendamos que en las estructuras temática e informativa se analizan las cláusulas propias del tema global y de la conexión informativa de la canción, y que en la estructura genérica, como se verá más adelante, se describen aquellos aspectos que son relevantes en la configuración formal del texto objeto de análisis.

\subsection{Análisis de la estructura genérica}

La tabla de la estructura genérica presenta la siguiente forma:

\begin{tabular}{|c|c|c|l|c|}
\hline Textura & \multirow{4}{*}{ Título del texto } \\
\cline { 3 - 4 } $\begin{array}{c}\text { Configuración } \\
\text { estructural }\end{array}$ & Intérprete & \multicolumn{2}{|c|}{ Función Narrativa } & $\begin{array}{c}\text { Sintaxis } \\
\text { narrativa }\end{array}$ \\
\cline { 3 - 5 } & & Evento & Participante \\
\hline
\end{tabular}

Las tablas anteriores se pueden aplicar a cualquier tipología textual, pero esta tabla en particular sólo es aplicable a textos cuya estructura genérica sea narrativa; y su orden sufre un cambio. La columna "Textura" aparece en primer lugar y se especifica en el término "Configuración estructural" ${ }^{2}$, que concierne a la organización formal del texto como canción y en la cual se presentan los mismos elementos existentes en cualquier muestra de la llamada música popular, pero con las denominaciones propias del aire musical en cuestión. En el caso de la champeta, los elementos que se analizan son: "Salutación" y/o "Presentación”, “Estrofas”, “Comentarios”, "Espeluque”, "Reiteraciones" y "Cierre".

La columna "Intérprete" pasa a la segunda posición y mantiene las características descritas anteriormente. La tercera columna, donde se anota el título de la canción como título de la tabla, es la que presenta la descripción en la estructura genérica. El motivo por el cual cambia el orden es porque intentamos analizar cómo, a partir de una forma básica, se organiza toda una narración, donde la secuencia funcional de eventos y participantes guarda las mismas propiedades lógicas del mundo natural que lo soporta.

Debajo de "Título de texto" se abren dos columnas: "Función narrativa" y "Sintaxis narrativa”. La primera alude a la función de los eventos y a los participantes en la organización lógico-semántica de los rela-

${ }^{2}$ Entendemos el término "configuración estructural" como la forma básica de los textos que se inscriben dentro del aire musical champeta, lograda a partir de ciertos elementos comunes que cumplen funciones estructurales dentro de ella (Ruíz, 2010:114). 
tos. Es decir, se describen aquellos segmentos donde, a partir de lo que se tematiza e informa en las cláusulas, se construye una función narrativa que integra una unidad lógica cuya base es la interrelación funcional entre eventos y participantes. En estos segmentos, por una parte, se representan participantes nominables según una dicotomía válida dentro de la temática supraordinal a la que se adhiere el texto, sea el amor, el engaño o la violencia. En este caso, la temática es la violencia (en una de sus variaciones, la violencia familiar), por lo que los participantes serán definidos en términos de "PerpetradorVictimario" y de "Víctima (implícita)" o "Víctima-Inhibido". Por otra parte, se describen eventos que son ejecutados por los participantes o que definen a éstos, y se entienden según su función en la organización de lo que se significa y se narra en torno a la temática supraordinal. Así, entonces, se habla de indicios caracterológicos, eventos funcional-cardinales y eventos funcional-catalizadores.

La segunda columna se refiere a la organización que se configura a partir de las funciones narrativas. Para el análisis se toma como base la forma de las narraciones cotidianas, esto es: Síntesis, Orientación, Resumen, Complicación, Resolución, Evaluación y Coda ${ }^{3}$. Estos elementos formales se constituyen con base en las funciones de los eventos y de los participantes. Veamos cómo se da el análisis de un segmento de una canción y, posteriormente, describámoslo someramente:

\begin{tabular}{|c|c|c|l|c|}
\hline Textura & \multirow{3}{*}{ Título del texto } \\
\cline { 3 - 4 } $\begin{array}{c}\text { Configuración } \\
\text { estructural }\end{array}$ & Intérprete & \multicolumn{2}{|c|}{ Función Narrativa } & $\begin{array}{c}\text { Sintaxis } \\
\text { narrativa }\end{array}$ \\
\cline { 3 - 5 } & & Evento & Participante \\
\hline
\end{tabular}

Como se puede observar, sí se tienen en cuenta elementos formales como el saludo. Y lo que hizo parte del tema -la trasposición adjetiva que permitía la caracterización del tema historia "de un mal padre"-, se convierte dentro la narración (funcionalmente) en indicio de algo:

\footnotetext{
${ }^{3}$ Según Labov, "Síntesis" corresponde a una o dos cláusulas que encierran el meollo de la narración y con la cual los narradores no intentan reemplazar el relato siguiente; la "Orientación" consiste en señalar los participantes, los eventos en los que ellos participan y las coordenadas espacio-temporales en que éstos ocurren; la "Complicación" alude al hecho de que hay ciertas acciones que en las narraciones desencadenan los eventos más importantes. La "Resolución" expresa la forma cómo se resuelve el problema introducido por la acción complicante ("Complicación"). Es decir, es una manera de cerrar una situación adversa en la historia. La "Evaluación" consiste en que el narrador indica la finalidad de la narración, el porqué lo contó y a dónde trata de llegar. Por último, la "Coda" se refiere a la forma cómo se cierra el texto y devuelve al interlocutor a su realidad (Lavob, 1988: 14-8).
} 
"abandonó a una niñita". Evento central, puesto que de él depende, lógicamente, la realización de otros eventos que funcionan como amplificadores de la información. Así, en esta organización lógica se evidencia un orden sintáctico que retoma ciertos valores de la realidad factual en que se desenvuelven los autores. Por ello el indicio "mal padre" es una evaluación, pues socialmente está mal visto que un padre abandone a sus hijos. Es lógico también comprender que una "niñita" resuelva la complicación del abandono con el llanto, pues es un proceso mental-conductual válido ante la tristeza que causa el hecho social en cuestión.

\subsection{Interpretación}

Después deorganizadaslas tablas deanálisis, lainformación contenida en ellas debe ser interpretada con base en una triangulación que consiste en establecer un diálogo entre 1) la perspectiva sistémico funcional, 2) la teoría pertinente a la temática supraordinal de las canciones objeto de análisis (en este caso, la teoría sobre la violencia familiar) y 3) las lecturas que hace el analista como estudioso de una ciencia (lingüística) y conocedor del fenómeno cultural en cuestión: un aire musical. La finalidad es lograr una interpretación lo más precisa posible, de tal modo que sea coherente con respecto a los universos significativos del enfoque lingüístico, la perspectiva teórica y el fenómeno estudiado. Podemos organizar tales interpretaciones en fichas artesanales o digitales donde se anoten los siguientes datos: estructura analizada, fecha de análisis, título de la canción, comentario y la cita pertinente.

Por ejemplo:

\section{Estructura temática}

"Camino a la delincuencia".

$06 / 08 / 2009$

Papomán.

Comentario: El autor, Papomán, presenta la historia como tema coherente con la predicación que le sigue. Se tematiza utilizando, como herramienta de orden, un deíctico demostrativo "Esta". Esto último convierte al aspecto tematizado en un tema textual. Es decir, la tematización se da por elementos estructurales como conjunciones, conectores o deícticos y apunta al orden o forma de presentación del discurso (Halliday, 2004: 79). 
Este tipo de fichas permite tener clara la información que hará parte del informe, las citas que lo sustentarán y la periodicidad con que se interpreta la información, pues es posible que se den variaciones, sea por haber leído cierta teoría antes no conocida o por haber encontrado otros datos más relevantes -no se deben hacer, por supuesto, fichas de las canciones por completo, sino de aquellos aspectos considerados pertinentes-. Después de contar con las fichas, las organizamos de acuerdo con la estructura analizada y por fecha. En otras palabras, separamos las de estructura temática, las de estructura informativa y las de estructura genérica: esto para percatarse de la continuidad interpretativa y de la coherencia entre el análisis de una estructura con respecto a las otras.

\subsection{Organización del informe}

Tras organizar las interpretaciones, procedemos a organizar el informe, conscientes de que un análisis lingüístico sin muestra del uso de la lengua recaba en la falsedad. Por lo mismo, tomamos las interpretaciones contenidas en las fichas, sustentándolas con apartes de las tablas de análisis (no necesitaremos todo el cuadro: no cabría en la hoja. Debemos tomar sólo los aspectos útiles). Para los ejemplos, el número de columnas es fijo, pero el de las filas de cláusulas es variable. Esto es: se toman cuatro columnas (fijo) y las filas de cláusulas necesarias. Veamos un ejemplo de cada estructura:

\section{Estructura Informativa:}

\begin{tabular}{|c|c|c|c|}
\hline Texto & $\begin{array}{l}\text { Estructura in- } \\
\text { formativa }\end{array}$ & $\begin{array}{c}\text { Coherencia li- } \\
\text { neal }\end{array}$ & $\begin{array}{c}\text { Cohesión in- } \\
\text { terna }\end{array}$ \\
\hline $\begin{array}{l}\text { Y ésta se quedó } \\
\text { solita acom- } \\
\text { pañando a la } \\
\text { madre } \\
\text { Dando gritos de } \\
\text { descuadre: }\end{array}$ & \multirow[t]{2}{*}{$\begin{array}{l}\text { Nuevo, estado } \\
\text { [quedó...] }\end{array}$} & \multirow{2}{*}{$\begin{array}{l}\text { Efecto de pro- } \\
\text { ceso conductual } \\
\text { [abandonó] }\end{array}$} & \multirow{2}{*}{$\begin{array}{c}\text { Conjunción, ex- } \\
\text { tensión, aditiva } \\
{[\mathbf{y}]}\end{array}$} \\
\hline $\begin{array}{l}\text { Y con su inocen- } \\
\text { cia: papito tenme } \\
\text { clemencia vente } \\
\text { conmigo a vivir }\end{array}$ & & & \\
\hline $\begin{array}{c}\text { Para no tener } \\
\text { que ir camino a } \\
\text { la delincuencia// }\end{array}$ & $\begin{array}{l}\text { Nuevo, acción } \\
\text { [no ir] }\end{array}$ & $\begin{array}{c}\text { Finalidad de } \\
\text { acción [dar grito } \\
\text { de descuadre] }\end{array}$ & $\begin{array}{l}\text { Frase preposicio- } \\
\text { nal [para] }\end{array}$ \\
\hline
\end{tabular}




\section{Estructura temática:}

\begin{tabular}{|c|c|c|c|}
\hline Texto & $\begin{array}{c}\text { Estructura te- } \\
\text { mática }\end{array}$ & $\begin{array}{c}\text { Cohesión in- } \\
\text { terna }\end{array}$ & $\begin{array}{c}\text { Realidades del } \\
\text { universo }\end{array}$ \\
\hline $\begin{array}{c}\text { Esta es la his- } \\
\text { toria de un mal } \\
\text { padre que aban- } \\
\text { donó a una niñita }\end{array}$ & $\begin{array}{c}\text { Tema Textual } \\
\text { [Historia] }\end{array}$ & $\begin{array}{c}\text { Referencia, deixis } \\
\text { demostrativa } \\
\text { [esta] }\end{array}$ & $\begin{array}{c}\text { Objeto de carac- } \\
\text { terización [his- } \\
\text { toria de un mal } \\
\text { padre] }\end{array}$ \\
\hline
\end{tabular}

\section{Estructura genérica:}

\begin{tabular}{|c|c|c|c|c|}
\hline \multirow{2}{*}{$\begin{array}{l}\text { Configura- } \\
\text { ción estruc- } \\
\text { tural }\end{array}$} & \multirow{2}{*}{ Texto } & \multicolumn{2}{|c|}{ Función narrativa } & \multirow{2}{*}{$\begin{array}{l}\text { Sintaxis } \\
\text { narrativa }\end{array}$} \\
\hline & & EV. & PAR. & \\
\hline \multirow{4}{*}{ Espeluque } & $\begin{array}{l}\text { Pégale los } \\
\text { cuerazos, } \\
\text { pégale los } \\
\text { cuerazos// }\end{array}$ & $\begin{array}{c}\text { Funcional, } \\
\text { Catalizador } \\
\text { [amplifica- } \\
\text { dor] }\end{array}$ & & \\
\hline & $\begin{array}{c}\text { A Juancho lo } \\
\text { vieron muy } \\
\text { triste y llo- } \\
\text { rando }\end{array}$ & $\begin{array}{l}\text { Funcional, } \\
\text { cardinal }\end{array}$ & $\begin{array}{l}\text { Víctima, } \\
\text { inhibido }\end{array}$ & \multirow{2}{*}{$\begin{array}{l}\text { Complica- } \\
\text { ción }\end{array}$} \\
\hline & $\begin{array}{l}\text { porque su } \\
\text { mujer le } \\
\text { estaba pe- } \\
\text { gando }\end{array}$ & $\begin{array}{c}\text { Funcional, } \\
\text { Catalizador } \\
\text { lógico [am- } \\
\text { plificador de } \\
\text { causa] }\end{array}$ & $\begin{array}{l}\text { Perpetrador, } \\
\text { Victimario }\end{array}$ & \\
\hline & $\begin{array}{l}\text { Pégale los } \\
\text { cuerazos// }\end{array}$ & $\begin{array}{c}\text { Funcional, } \\
\text { Catalizador } \\
\text { [amplifica- } \\
\text { dor] }\end{array}$ & & \\
\hline & $\begin{array}{l}\text { Se creía un } \\
\text { traga cancha }\end{array}$ & & & \multirow[b]{2}{*}{ Evaluación } \\
\hline & $\begin{array}{c}\text { mujeres ma- } \\
\text { las, Con esa } \\
\text { mujer se te } \\
\text { ve vejez }\end{array}$ & & & \\
\hline
\end{tabular}

\section{Conclusiones}

Con el análisis de cada sistema del componente textual se obtienen ciertos resultados que giran alrededor del descubrimiento de aquellos aspectos ideológicos que subyacen en el texto como parte "visible" del todo semiótico del aire musical en cuestión. En primer lugar, al analizar el sistema temático, el investigador pretende descubrir el aspecto de la realidad factual (participantes, eventos, 
herramientas o circunstancias espaciales y temporales) al que se da mayor estatus temático en el conjunto de textos tomado como objeto de estudio. En otras palabras, analizar temáticamente la letra de una canción implica dilucidar aquella unidad experiencial que el escritor selecciona como unidad digna de ser significada y digna de recibir la predicación que se desarrolla como rema en la secuencia de significado del texto (Halliday, 2004: 65).

Ahora bien, a este resultado no se debe llegar por la mera lectura de las cláusulas del texto, sino que la cohesión interna de las clausulas que lo integran debe llevar a los descubrimientos señalados anteriormente. Por ello, analizar el sistema temático es también estudiar los recursos textuales que el escritor selecciona del conjunto de opciones que le ofrece su lengua, para expresar sus concepciones sobre el mundo. Al respecto, Halliday afirma que "en el habla es evidente que los recursos fonológicos son bien marcados en la expresión de la información, pero en el ámbito de lo escrito no se cuenta con este aspecto, por lo cual hay que recurrir a elementos de la expresión escrita (elipsis, conectores, correferencias, etc.)" (1982: 175).

Por otro lado, al analizar el sistema informativo, el investigador pretende 1) descubrir las informaciones que se presentan como noticia con respecto a las unidades experienciales que han sido ubicadas en estatus temático; 2) identificar las relaciones semánticas que se configuran, y 3) encontrar la conexión informativa ${ }^{4}$ que existe entre esas nuevas informaciones. Con el hallazgo de los tres elementos mencionados comprendemos cuáles son los conocimientos culturales que el autor intenta expresar y cómo las relaciones entre los eventos que se cuentan responden positiva o negativamente a la lógica de los eventos en la realidad factual, hasta el punto de que se pueden representar a partir de una coherencia lineal, cuya base son las relaciones semánticas (Van Dijk, 1996: 25). Son precisamente estas relaciones semánticas las que configuran

\footnotetext{
${ }^{4}$ Definimos "conexión informativa" como una red o entretejido de información donde una nueva información conlleva y justifica a otra. Dicha red se compone de varios nodos informativos (segmentos en los que confluyen intercesiones semánticas organizadas no jerárquicamente para configurar el texto como una unidad de significados) (Ruiz, 2010: 73). De igual forma, se explica que en el esquema de conexión informativa las llaves denotan la regencia y dependencia de una información sobre otra. En la parte cerrada se ubica el regente y en la parte abierta, el dependiente. En la llave mayor se ubica el nodo de información y en las menores se ubican los recursos no estructurales de la coherencia lineal que componen cada nodo. Con las flechas se indican las informaciones nuevas contenidas en cada recurso no estructural. Con el arco se expresa cuándo una relación semántica da apertura a un nuevo nodo. Igualmente, cuándo de todo un nodo se abre otro (79).
} 
una tensión alrededor de lo conocido y lo nuevo, entre lo dado anteriormente y lo que se presenta como una noticia con el fin de dar una lógica de ampliación al mensaje que se está construyendo en el texto como unidad semántica (Halliday, 1985: 91). Veamos a continuación un gráfico de la conexión informativa:

\section{Esquema de conexión informativa}

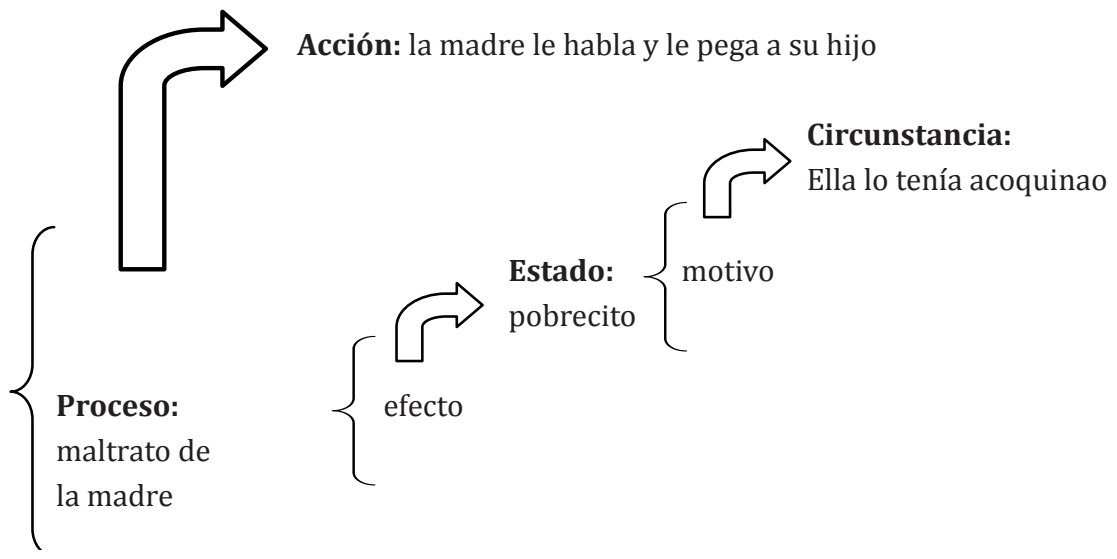

Porúltimo, con elanálisis dela estructuragenéricaseintenta descubrir la "configuración estructural" de las canciones de champeta, para luego entender cómo a partir de ella se evidencia una lectura sobre un aspecto de la sociedad, es decir, la macrodefinición que sobre la temática supraordinal se evidencia (incluyendo las definiciones particulares) como resultado de la simbolización de un mundo ficcional realista. Macrodefinición que se obtiene con el análisis funcional de los eventos organizados en la estructura genérica del texto que, teóricamente, parte de una convención de ficcionalidad en la que es observado como el resultado de un proceso semiótico por el cual la significación plena y el efecto estético sólo se producen cuando es leído con referencia al mundo ficcional creado en él.

Así entonces, tanto autores como oyentes deben ser conscientes de que los referentes del mundo real están suspendidos y son sustituidos por los mecanismos de referencia al mundo ficcional que acordaron en considerar como verdadero y real (Paz Gago, 1989: 107-8). Esto último, efectivamente, permite afirmar que en las canciones de un aire musical se puede exponer un correlato del mundo real que, sin ser una copia exacta de éste, permite que los eventos narrados en el texto tengan las mismas propiedades que los eventos en el mundo real, incluyendo su lógica causal y racional (Paz Gago, 1989: 109). 


\section{Bibliografía}

Barthes, R. (1989). "Introducción al análisis estructural de los relatos". En Niccolini, S. (1977). El análisis estructural. Buenos Aires: Centro Editor de América Latina.

Halliday, M. (1985). El lenguaje como semiótica social: la interpretación social del lenguaje y del significado. México: Fondo de Cultura Económica.

(2004). An Introduction to Functional Grammar (3ํㅡ. Ed.). New York: Oxford University Press.

Labov, W. (1988). La transformación de la experiencia en sintaxis narrativa. Cali: Universidad del Valle.

Paz Gago, J. (1989). Semiótica del Quijote: Teoría y práctica de la ficción narrativa. Barcelona: Emecé.

Ruíz Toro, D. (2010). Análisis lingüístico-funcional del componente textual en canciones de champeta sobre violencia familiar. Tesis de Maestría en Lingüística. Universidad Nacional de Colombia, Bogotá.

Van Dijk, T. (1996). Estructuras y funciones del discurso. México: Siglo XXI Editores. 
